\title{
MANEJO QUIRÚRGICO DEL CÁNCER DE PIEL SERVICIO DE DERMATOLOGÍA, HOSPITAL DE SAN JOSÉ, BOGOTÁ DC, COLOMBIA
}

\author{
Oscar Mora H. MD*, Manuel Fernando Ortíz MD**, Angélica Guatibonza H. MD***, \\ Itala Merlano Caropresse MD***
}

\section{Resumen}

Introducción: el cáncer de piel representa un problema de salud pública que se puede prevenir y manejar con éxito ante el diagnóstico temprano. Objetivo: describir las características clínicas, histopatológicas, poblacionales y quirúrgicas del cáncer de piel tratado mediante cirugía en el Hospital de San José de Bogotá DC, de enero 2009 a diciembre 2010. Material y métodos: estudio descriptivo y retrospectivo de dos años de los casos confirmados de carcinoma basocelular, escamocelular y melanoma in situ. Resultados: de 294 historias clínicas revisadas se tomaron cien con diagnóstico de cáncer cutáneo que cumplieron con los criterios de inclusión: 84 pacientes $(84 \%)$ con carcinoma basocelular, trece $\mathbf{( 1 3 \% )}$ ) con carcinoma escamocelular y tres $(\mathbf{3} \%)$ con melanoma in situ. Conclusión: con el presente trabajo presentamos las normas del manejo quirúrgico de los pacientes con cáncer de piel en un hospital general.

Palabras clave: carcinoma basocelular, carcinoma escamocelular, melanoma in situ.

Abreviaturas: CBC, carcinoma basocelular; CEC, carcinoma escamocelular, MIS, melanoma in situ.

\section{SURGICAL MANAGEMENT OF SKIN CANCER, DERMATOLOGY DEPARTMENT, HOSPITAL DE SAN JOSÉ, BOGOTÁ DC, COLOMBIA}

\begin{abstract}
Introduction: skin cancer is a public health problem which may be prevented and treated successfully if diagnosed early. Objective: to describe the clinical, hystopathologic, demographic and surgical characteristics of surgically treated cases of skin cancer at Hospital de San José, Bogotá DC, from January 2009 to December 2010. Material and Methods: a two-year descriptive retrospective study of confirmed cases of basal cell carcinoma, squamous cell carcinoma and melanoma in situ. Results: one hundred cases of diagnosed skin cancer which met inclusion criteria were selected from 294 clinical records reviewed: 84 patients $(84 \%)$ had basal cell carcinoma (BCC), thirteen (13\%) had squamous cell carcinoma (SCC) and three (3\%) had melanoma in situ. Conclusion: we hereby present surgical management guidelines for patients with skin cancer at a general hospital.
\end{abstract}

Key words: basal cell carcinoma, squamous cell carcinoma, in situ melanoma.

Fecha recibido: diciembre 13 de 2012 - Fecha aceptado: marzo 18 de 2013

* Dermatólogo, Hospital de San José. Coordinador de la especialización en dermatología. Instructor Asistente, Fundación Universitaria de Ciencias de la Salud. Bogotá DC, Colombia.
** Residente III de Dermatología. Fundación Universitaria de Ciencias de la Salud. Bogotá DC, Colombia.

*** Residente II de Dermatología. Fundación Universitaria de Ciencias de la Salud. Bogotá DC, Colombia. 


\section{Introducción}

La Asociación Americana de Cáncer $(A A C)$ estableció que cerca de 1 a 1,3 millones de casos de cáncer de piel, excluyendo los melanomas, se diagnostican en el año en Estados Unidos, de los cuales 50\% a $65 \%$ son CBC y $20 \%$ a $25 \%$ CEC. ${ }^{1}$ Las Naciones Unidas han estimado que en el mundo ocurren 200.000 casos por año de melanoma cutáneo y más de dos millones de otros tipos de cáncer de piel. ${ }^{2}$ Australia es uno de los países con la tasa más alta de cáncer cutáneo debido a los altos niveles de radiación ultravioleta proveniente de los rayos solares, combinado con una alta susceptibilidad de la piel blanca en esta población. Así, de los veinte millones de habitantes más de 1.700 australianos mueren al año por esta causa. ${ }^{3,4}$

En Colombia la incidencia aumentó de 23 casos por cada 100.000 habitantes en 2003, a 41 en 2007. La prevalencia en ambos sexos fue igual y la proyección de incidencia en el país hasta el 2020 alcanza una tasa esperada de102 casos/100.000 habitantes, de acuerdo con la observación realizada por Sánchez y col. ${ }^{5} \mathrm{El}$ Hospital de San José con un largo historial asistencial y científico, reconocido como una de las mejores instituciones médicas del país y su servicio de dermatología con experiencia de más de 30 años, atiende un importante número de pacientes con cáncer cutáneo, lo que motivó nuestro interés en realizar el presente estudio.

\section{Métodos}

Se realizó un estudio descriptivo de serie de casos; se incluyeron pacientes con diagnóstico confirmado de CBC, CEC y MIS, tratados mediante cirugía en el período comprendido desde enero 2009 hasta diciembre 2010, en el Hospital de San José, Bogotá DC. Se excluyeron los tratados con otros métodos (electrocuretaje, criocirugía, imiquimod tópico y aplicación de 5-fluorouracilo) y aquellos con diagnóstico de otro tipo de melanoma cutáneo o lesiones premalignas. La información se obtuvo de las historias clínicas del servicio de estadística del hospital y los datos registrados se ingresaron enuna base de datos en excel. Se examinaron variables poblacionales, histopatológicas, clínicas y quirúrgicas, que de acuerdo con su naturaleza se analizaron utilizando medidas de tendencia central, de dispersión, frecuencias absolutas y relativas, utilizando la base de datos STATA 10.

\section{Resultados}

De los cien pacientes con diagnóstico de cáncer de piel que recibieron tratamiento quirúrgico, $84 \%$ (84/100) fueron diagnosticados como CBC, $13 \%$ (13/100) CEC y $3 \%$ (3/100) MIS. El 47\% fueron hombres y la edad promedio fue de 68,5 años, (rango 41-87 años). El tiempo mediano transcurrido para el diagnóstico registrado en las historias clínicas fue para el CBC doce meses (6-24), CEC 18 meses (12-48) y para MIS 10 meses (6-24) (Figura 1).

El 57,1\% de los CBC fueron encontrados en la zona de alto riesgo o zona $\mathrm{H}$ de la cara que incluye las regiones centrofacial, periorbitaria inferior, temporal, pre y retroauricular. ${ }^{6}$ Los sitios anatómicos del CEC fueron región centrofacial 23\% (3/13), piel cabelluda 23\% (3/13), extremidades $23 \%$ (3/13), tronco15\% (2/13) y pabellón auricular 7,6\% (1/13). Los tres casos de MIS estaban localizados en la cara. Los patrones histopatológicos en CBC fueron 58 (69,8\%) nodular, 5 (6\%) superficial, $5(6 \%)$ trabecular, $1(1,2 \%)$ micronodular y $12(14,4 \%)$ mixto.

Con respecto a la diferenciación histológica para el CEC encontramos cinco (38\%) como mal diferenciados, tres $(23 \%)$ bien y $2(15 \%)$ moderados. En tres (23\%) no hubo registro. En cuanto al compromiso perineural nueve (69\%) no lo presentaban, uno $(7,6 \%)$ se confirmó y en tres $(23 \%)$ no se encontró información.

El grupo de pacientes incluidos en nuestro estudio fue manejado mediante resección local siguiendo las guías del Instituto Nacional de Cancerología y las del NCCN (National Comprensive Cancer Network) ${ }^{6-7}$ La reconstrucción del defecto quirúrgico se realizó mediante las siguientes técnicas: cierre primario, colgajo, injerto y cierre por segunda intención, considerando el tamaño y el área anatómica. Los hallazgos histopatológicos posoperatorios en los casos de $\mathrm{CBC}$, mostraron que en $70(83 \%)$ se encontraban bordes libres de tumor, siete $(8,3 \%)$ con compromiso de uno de los bordes 


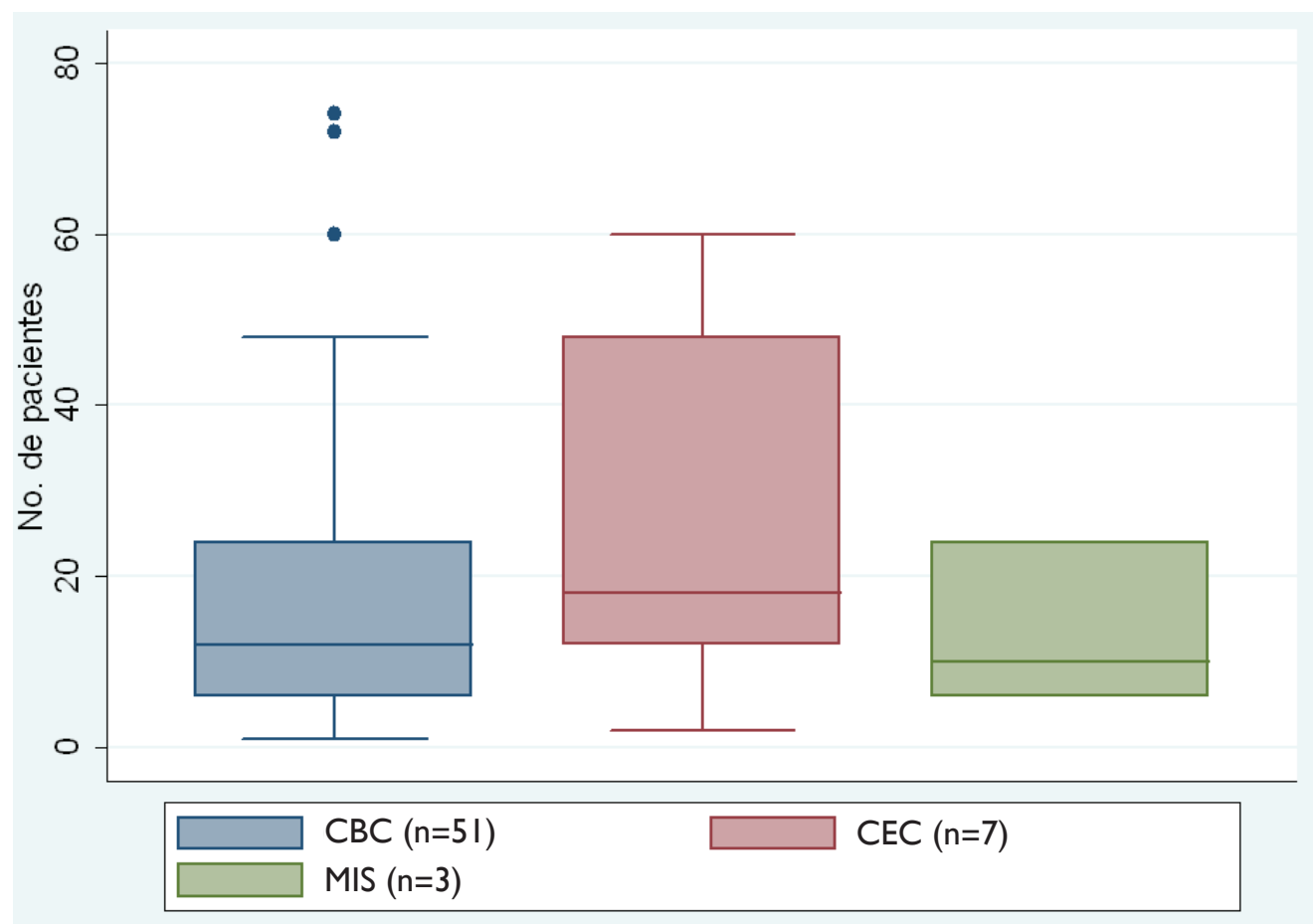

Figura I. Cajas del tiempo de evolución.

de sección y siete pacientes $(8,3 \%)$ no asistieron al control. En CEC siete $(53,8 \%)$ presentaron márgenes libres de tumor, uno $(7,6 \%)$ con compromiso y cinco $(38,4 \%)$ no asistieron a control.

Los tres pacientes con diagnóstico de melanoma fueron resecados por completo encontrándose los bordes libres de tumor, dos de ellos con manejo multidisciplinario por los servicios de otorrinolaringología y cirugía de cabeza y cuello. No se encontraron complicaciones intraoperatorias. En las posoperatorias $10 \%$ presentó alteraciones de la cicatrización como queloides y cicatriz hipertrófica.

\section{Discusión}

En nuestro servicio encontramos que el CBC nodular se presenta en un mayor número de pacientes, el tratamiento dado fue la cirugía convencional y el cierre primario la técnica de reconstrucción más utilizada (Tabla 1), en coherencia con lo revisado en la literatura, según la cual el tipo de cáncer más frecuente es el CBC con incremento progresivo de su incidencia,siendo el tratamiento propuesto la escisión local amplia, con una tasa de curación del 90\%.5,11,12

\begin{tabular}{|c|c|c|}
\hline Técnicas de reconstrucción & $n=100$ & (\%) \\
\hline \multicolumn{3}{|l|}{ СВС } \\
\hline colgajo & 20 & $(23.5)$ \\
\hline injerto & 2 & $(2.4)$ \\
\hline cierre primario & 58 & $(69.4)$ \\
\hline cierre secundario & 4 & (4.7) \\
\hline \multicolumn{3}{|l|}{ CEC } \\
\hline colgajo & 3 & (23) \\
\hline cierre primario & 10 & (77) \\
\hline \multicolumn{3}{|l|}{ MIS } \\
\hline cierre primario & 3 & $(100)$ \\
\hline
\end{tabular}

Los patrones histológicos y su localización en la zona $\mathrm{H}$ en orden de frecuencia fueron: nodular, mixto, trabecular, superficial y mixto, compuesto por dos o más tipos histológicos. ${ }^{12,13}$ Una limitante fue no considerar en todos los casos el compromiso perineural.

Con respecto al CEC ocupa el segundo lugar de los cánceres de piel en nuestro servicio. La ubicación anatómica más frecuente es cara, manos y brazos, pero no podemos relacionar en forma directa esta situación 
que si bien se encuentra acorde con lo revisado en la literatura, puede variar debido a características propias de nuestra población como el fototipo de piel y la ocupación (exposición constante a los rayos solares). ${ }^{14,15}$

Los tres MIS corresponden a léntigo maligno localizados en la zona facial teniendo en cuenta los criterios de inclusión. Es importante resaltar que el tipo de melanoma cutáneo más frecuente en nuestro país es el lentiginoso acral, el cual no hizo parte de los criterios de inclusión.

También se encontró que no hubo diferencia de presentación del CBC, el CEC y melanoma con respecto al género; la edad promedio de diagnóstico fue 68,5 años y en el cáncer de piel no melanoma, el promedio de edad de diagnóstico registrado para los hombres está en un rango entre 59 a 65 años y para las mujeres de 60 a $66 .{ }^{16}$

Acorde con la literatura nuestros hallazgos de manejo posescisión local amplia ${ }^{11}$, encontramos que en la mayoría la reconstrucción fue cierre primario, dejando un pequeño grupo para reparación mediante colgajo o injerto y unos pocos para cierre por segunda intención. Las técnicas reconstructivas van encaminadas a brindar funcionalidad y buen aspecto estético al defecto quirúrgico después de una cirugía, para lo cual se tienen en cuenta las subunidades faciales donde se localice así como el tamaño, lo cual determina la técnica reconstructiva apropiada para cada paciente. ${ }^{17}$

El tiempo promedio de diagnóstico fue de más de diez meses, llamando la atención en especial para el CEC y MIS, entidades de peor pronóstico donde el diagnóstico temprano es valioso para el tratamiento oportuno. Proponemos con estos hallazgos una mayor educación tanto a nivel de pre y posgrado, al igual que generar en nuestros pacientes cultura de fotoprotección y consulta oportuna a su médico dermatólogo ante la presencia de cualquier lesión sugestiva de tumor. Las complicaciones fueron escasas, tardías y ninguna intraoperatoria, como la formación de queloide y cicatriz hipertrófica que depende en gran medida de la ubicación de la lesión y la idiosincrasia propia del paciente. ${ }^{18}$ Con respecto al compromiso de los bordes de disección en CBC y CEC, se concluyó que se debe en gran medida al patrón histológico disperso del tumor y los bordes clínicos mal definidos de la lesión.

Los datos sobre los cuales nos basamos para elaborar el trabajo fueron extraídos de las historias clínicas del servicio, encontrando limitantes por información insuficiente consignada como fototipo de piel y ocupación del paciente, donde se especifique el antecedente de exposición solar. Teniendo en cuenta que es un estudio descriptivo retrospectivo no nos permite evaluar el pronóstico y seguimiento. Proponemos realizar estudios en otros hospitales de dermatología general para conocer la incidencia real en el país y confirmar la tendencia actual del ascenso del cáncer cutáneo en Colombia.

\section{Referencias}

1. American Cancer Society. Cancer facts and figures 2003. Learn about cancer[monografía en Internet]. Clifton Roa, NE: ACS; 2003. [citado22 Mar. 2013].Disponible en:http://www.cancer.org/research/cancerfactsfigures/cancerfactsfigures/index.

2. Belkin M, Césarini P, Diffey B, Hietanen M, Kojima M, Mariutti G, et al. Protection against exposure to ultraviolet radiation. Geneva: WHO; 1995. [citado 22 Mar. 2013]. Disponible en:http://www.who.int/uv/publications/proUVrad.pdf.

3. Sinclair C, Foley P. Skin cancer prevention in Australia. Br J Dermatol. 2009 Nov; 161Suppl 3:116-23.

4. Makin J. Implications of climate change for skin cancer prevention in Australia. Health Promot J Austr. 2011. Dec: 22 Spec No: S39-41.

5. Sanchez G., Nova J., De la Hoz F., Castañeda C. Incidencia de cáncer de piel en Colombia, años 2003-2007. Piel. 2011;26(4):171-7.

6. Rueda X, Acosta de Hart A, Aristizabal L, Fierro E. Guías de práctica clínica para el tratamiento del carcinoma basocelular. Rev. Col Dermatol. 2008; 16. (2): 102-17.

7. Rueda X, Acosta de Hart A, Alba C, Pulido L. Guías de práctica clínica para el tratamiento del carcinoma escamocelular. Rev. Col Dermatol. 2008; 16. (2): 116-134.

8. National Comprehensive Cancer Network. Clinical Practice Guidelines in oncology: guidelines index basal cell and squamous cell TOC staging. Washington: NCCN; 2006

9. Balch DM, Soong S, Ross MI, et al. Long term results of a multi-institucional randomized trial comparing prognostic factors and surgical results for intermediate thieckness melanomas (1,0 to $4,0 \mathrm{~mm})$. Intergroup Melanoma Surgical Trial. Ann Surg Oncol. 2000:7(2):87-97.

10. Kunishige JH, Brodland DG, Zitelli JA. Surgical margins for melanoma in situ. J Am Acad Dermatol. 2012;66(3):438-44.

11. Ho T, Byrne PJ. Evaluatioin and initial management of the patient with facial skin cancer. Facial Plast.Surg. Clin North Am. 2009. Aug: 17(3):301-7.

12. Sánchez G, Nova J, De la Hoz F. Factores de riesgo de carcinoma basocelular. Un estudio del Centro Nacional de Dermatología de Colombia. Actas Dermosifiliogr. 2012;103(4):294-300.

13. Firnhaber J. Diagnosis and treatment of basal cell and squamous cell carcinoma. Am FamPhysician. 2012 Jul 15:86(2):161-8.

14. Netscher DT, Leong M, Orengo I, Yang D, Berg C, Krishnan B. Cutaneous malignancies: melanoma and nonmelanoma types. Plast.Reconstr.Surg. 2011. Mar:127(3):37e.56e.

15. Nova J, Sánchez G, Peña E. Características epidemiológicas de pacientes con carcinoma escamo celular cutáneo en el Centro Dermatológico Federico Lleras Acosta, Bogotá, Colombia. Rev. Asoc.Colomb Dermatol. 2011;19: 212-17.

16. Kim R, Armstrong A. Nonmelanoma skin cáncer. Dermatol Clin.2012 Jan.; 30(1): 125-39,

17. Hofer SO, Improving outcomes in aesthetic facial reconstruction.Clin. Plast. Surg. 2009 Jul.; 36(3): 345-54

18. Jiménez, Vázquez B, Gómez G, Moreno G. Complicaciones en dermatología quirúrgica. Actas Dermosifiliogr. 2009; 100:661-8. 